\title{
Short-term outcomes in patients undergoing laparoscopic surgery for deep infiltrative endometriosis with rectal involvement: a single-center experience of 168 cases
}

\author{
Sara Gortázar de las Casas ${ }^{1, \star}$, Emanuela Spagnolo $^{2, \star}$, Salomone Di Saverio $^{3}$, Mario Álvarez-Gallego $^{1}$, \\ Ana López Carrasco ${ }^{2}$, María Carbonell López ${ }^{2}$, Sergio Torres Cobos ${ }^{4}$, Constantino Fondevila Campo ${ }^{1}$, \\ Alicia Hernández Gutiérrez ${ }^{2}$, Isabel Pascual Miguelañez ${ }^{1}$ \\ ${ }^{1}$ Department of General Surgery, La Paz University Hospital, Madrid; ${ }^{2}$ Department of Obstetrics and Gynecology, La Paz University Hospital, \\ Madrid, Spain; ${ }^{3}$ Department of General Surgery, Hospital of San Benedetto del Tronto (AP), San Benedetto, Italy; ${ }^{4}$ Department of Stoma \\ Therapy, La Paz University Hospital, Madrid, Spain
}

Purpose: The surgical management of deep infiltrative endometriosis (DE) involving the rectum remains a challenge. The objective of this study was to assess the outcomes from a single tertiary center over a decade with an emphasis on the role of a protective loop ileostomy (PI).

Methods: A retrospective review of outcomes for 168 patients managed between 2008 and 2018 is presented including 57 rectal shaves, 23 discoid excisions, and 88 segmental rectal resections.

Results: The nodule size (mean \pm standard deviation) in the segmental resection group was $32.7 \pm 11.2 \mathrm{~mm}, 23.4 \pm 10.5$ $\mathrm{mm}$ for discoid excision, and $18.8 \pm 6.0 \mathrm{~mm}$ for rectal shaves. A PI was performed in 19 elective cases (11.3\%) usually for an ultra-low anastomosis $<5 \mathrm{~cm}$ from the anal verge. All Clavien-Dindo grade III/IV complications occurred after segmental resections and included 5 anastomotic leaks, 6 rectovaginal fistulas, 2 ureteric fistulas, and 1 ureteric stenosis. Of 26 stomas (15.5\%), there were 19 PIs, 3 secondary ileostomies (after complications), and 4 end colostomies. The median time to PI closure was 5.8 months (range, 0.4-16.7 months) in uncomplicated disease compared with 9.2 months (range, 4.7-18.4 months) when initial postoperative complications were recorded $(\mathrm{P}=0.019)$. Only 1 patient with a recurrent rectovaginal fistula had a permanent colostomy.

Conclusion: In patients with DE and rectal involvement a PI is selectively used for low anastomoses and complex pelvic reconstructions. Protective stomas and those used in the definitive management of a major postoperative complication can usually be reversed.

Keywords: Endometriosis; Laparoscopic surgery; Ileostomy; Colostomy

Received: Sep 23, 2021 - Revised: Oct 28, 2021 - Accepted: Nov 6, 2021

Correspondence to: Salomone Di Saverio, M.D., Ph.D, FACS, FRCS (Eng)

General Surgery Unit, San Benedetto del Tronto Hospital, AV5, ASUR

Marche, San Benedetto del Tronto, Italy

Tel: +39-3486007338

E-Mail: salo75@inwind.it

ORCID: https://orcid.org/0000-0001-5685-5022

Corresponding author's current affiliation is Department of General and Specialized Surgery "Paride Stefanini", La Sapienza University of Rome, Rome, Italy.

* Sara Gortázar de las Casas and Emanuela Spagnolo contributed equally to this work as co-first authors.

(C) 2022 The Korean Society of Coloproctology

This is an open-access article distributed under the terms of the Creative Commons Attribution NonCommercial License (https://creativecommons.org/licenses/by-nc/4.0) which permits unrestricted noncommercial use, distribution, and reproduction in any medium, provided the original work is properly cited.

\section{INTRODUCTION}

Deep infiltrative endometriosis (DE) is a benign but aggressive disease, predominantly affecting premenopausal women [1]. It is defined by the presence of endometrial tissue outside of the uterus, invading more than $5 \mathrm{~mm}$ of the peritoneal surface [2]. Rectal involvement in endometriosis has a prevalence ranging between $5.3 \%$ and $12 \%$ [3] with the rectum and the sigmoid colon accounting for more than $90 \%$ of gastrointestinal DE cases [4]. The surgical excision of endometriosis is indicated after failure of medical treatment and when lesions become symptomatic by im- 
pairing bowel, urinary, sexual, and reproductive functioning [5]. In cases with rectal involvement, there are 3 main surgical options depending upon the degree of rectal wall infiltration and the size of the endometriotic nodule. These alternatives include rectal shaving, discoid resection, and segmental rectal resection [6]. A protective loop ileostomy (PI) can be selectively performed en passant with segmental rectal resection in order to reduce the consequences of an anastomotic leak [7]. There remains debate, however, concerning the indications for a PI and its effect on functional outcome [8]. We present a 10-year, single surgical unit experience with stoma use in the management of colorectal endometriosis.

\section{METHODS}

\section{Ethics statement}

Permission for the conduct of a retrospective survey was provided by the La Paz Hospital ethics committee (No. PI-3349). All procedures performed in studies involving human participants were in accordance with the ethical standards of the institutional and/or national research committee and with the 1964 Helsinki declaration and its later amendments or comparable ethical standards. Informed consent was obtained from all individual participants included in the study.

\section{Subjects participated}

Analysis was conducted from a surgical database derived from patients managed with DE plus rectal involvement who presented between January 2008 and December 2018 at the Hospital Universitario La Paz, Madrid, a 1,300-bed tertiary referral center. Patients were registered on a dedicated prospectively accumulated database and cross-checked with ward and surgery registers and pathology reports. Inclusion criteria for analysis were all adults aged $>18$ years who were surgically managed by the gynecology department for endometriosis with rectal involvement. For the purposes of definition, this included endometriotic invasion of the rectal wall with a location extending up to $15 \mathrm{~cm}$ above the anal margin. Cases of DE without bowel involvement were excluded from analysis.

Demographic data were collated along with the length of hospital stay (LOHS). Information gathered included a history of prior surgeries, procedures performed specifically for endometriosis, operative time, associated surgical procedures, and the use of stomas. Complications following endometriosis surgery were registered in accordance with the Clavien-Dindo classification [9]. For the purposes of definition, protective or primary stomas were defined as those performed during elective surgery with the view that their preemptive construction would reduce the local and systemic consequences (rather than the likelihood) of an anastomotic leak. All stomas in this group were PIs. Stomas were defined as secondary if they were performed in specific scenarios most notably in an urgent setting or due to complications after elective surgery (anastomotic leak, rectovaginal fistula). Secondary stomas included either a secondary loop ileostomy (SI) or a terminal colostomy fashioned during a Hartmann style procedure. The type of ostomy (primary or secondary), the time until stoma closure, and testing performed in order to verify the integrity of the colorectal anastomosis or the integrity of the vaginal and/or rectal wall were recorded along with any complications associated with stoma closure.

\section{Workup and surgical technique}

At our institution, all DE cases are discussed in a multidisciplinary committee comprised of gynecologists, urologists, and colorectal surgeons, all of whom are experienced in complex minimally invasive pelvic surgery. All of the operations were performed by consultants from this combined endometriosis unit. Each patient had a preliminary outpatient preoperative consultation visit with the team of surgeons in order to plan surgery along with recording of the findings of a preoperative transvaginal ultrasound and/ or pelvic magnetic resonance imaging. When rectal wall infiltration was suspected, a preoperative consultation with the stoma therapist for stoma education and stoma site marking was scheduled. Patients routinely underwent bowel preparation with polyethylene glycol and oral antibiotics (metronidazole and neomycin). Operations were covered with intravenous perioperative antibiotics (amoxicillin-clavulanic acid or gentamicin and ciprofloxacin in allergic patients) in accordance with hospital protocols. Operative procedures performed concomitantly with the rectal surgery included adhesiolysis, ovarian cystectomy, excision of other endometriotic implants, adnexectomy, and hysterectomy. In recent years, the ureters are cannulated at cystoscopy with installation of $8 \mathrm{~mL}(1.25 \mathrm{mg} / \mathrm{mL})$ of indocyanine green (ICG) prior to laparoscopy for infrared ureteral fluorescence to identify the ureter and any ureteric endometriosis [10].

During surgery, bowel lesions are evaluated, noting the size of nodules, the presence of strictures, and the extent of infiltration. Depending upon each factor, decision is made for 1 of 3 management techniques. All pelvic procedures were performed with autonomic nerve preservation. Shaving is performed when an endometriotic lesion infiltrates from the peritoneum to the muscularis of the rectal wall. In this circumstance, scissor excision is used rather than coagulation diathermy so as to separate the nodule from the rectum in the correct plane of cleavage and in order to avoid the risk of mucosal perforation [5]. In cases with deeper infiltration of the rectal wall and for nodules that are $<3 \mathrm{~cm}$, a disc (full thickness) excision is performed typically using a 31-mm circular stapler. A 3/0 vicryl suture is inserted into the endometriotic nodule so that it can be interposed between the anvil and a transanally introduced stapler. The stapler is then closed and fired, performing excision of the anterior wall of the rectum which included the endometriotic nodule.

Segmental resection is reserved for cases with larger nodules ( $>3 \mathrm{~cm}$ ), those with $>40 \%$ stenosis of the lumen and for multiple 
lesions. As DE is a benign condition, resections are performed with only a partial mesorectal excision. After detaching the nodule from surrounding structures, the rectum is transected distally with a $60-\mathrm{mm}$ linear stapler leaving a $1-\mathrm{cm}$ margin of healthy tissue. The rectum is then exteriorized through a Pfannenstiel abdominal wall incision performing the proximal transection after which the anvil of the circular stapler can be fitted into place. When a hysterectomy is also being performed the rectum can be brought through the vaginal stump so that a laparoscopic colorectal anastomosis using the circular stapler can then be performed $[5,11]$. The integrity of the anastomoses was assessed with either a standard pneumatic test (where the pelvis was filled with warm saline and the rectum insufflated with air) or where the rectum was instilled with methylene blue (Michelin test).

We recorded the type of resection according to the distance from the anal verge such that it was considered ultra-low if $<5$ $\mathrm{cm}$ and low at 5 to $8 \mathrm{~cm}$ [11]. The decision for PI in these cases depended upon the level of the anastomosis with diversion routine for ultra-low cases. In the event of a higher anastomosis, a PI was elected depending upon the intraoperative findings, the need for a complex ancillary urinary procedure, or when there were other concomitant systemic risk factors such as significant anemias or hypoalbuminemia. The PI was fashioned by identifying the ileal loop and delivering it through a disc skin excision over the site marked preoperatively. The ileum was brought through the abdominal wall ensuring that it was not rotated and that the afferent limb was positioned superiorly. The PI was matured after closure of the abdominal wall by an eccentric incision that ensured a 1 to $2-\mathrm{cm}$ eversion of the afferent limb. An anastomotic leak was defined as a defect in the bowel wall at the colorectal anastomosis leading to communication of the intra- and extraluminal compartments. A leak was identified either by computed tomography (CT) or at repeat surgery [12].

In our unit, routine blood testing of C-reactive protein (CRP) is performed on the 2 nd and 4 th postoperative days [13]. A progressive elevation of the CRP level provides an early warning sign of the potential emergence of a complication although it is a nonspecific test. In suspicious cases, an emergency CT scan was requested so as to search for an anastomotic complication. After an anastomotic leak was diagnosed, its severity was assessed where depending upon the attendant clinical symptoms, the blood results, and the radiological findings; the decision was made for either conservative or surgical management. Where the patient was clinically stable with a contained leak (with either an inflammatory phlegmon or a collection of $<3 \mathrm{~cm}$ ), bowel rest and broadspectrum antibiotics were used with continued serial abdominal and clinical examination evaluating for signs of free perforation [14]. Emergency surgery was performed in unstable patients as well as in those with signs of diffuse peritonitis or if there was failed conservative management. The principles of surgery relied upon peritoneal lavage and drainage plus a secondary ostomy as needed. A SI was fashioned for a small anastomotic leak without fecal peritonitis and a terminal colostomy (Hartmann procedure) was performed in those cases with a large anastomotic leak and diffuse fecal peritonitis. Stomal closure was scheduled depending upon resolution of the sequelae of the anastomotic leak [15]. Prior to closure, the integrity of the rectal and vaginal walls was confirmed with a combination of barium studies, repeat CT and endoscopy.

\section{Statistical analysis}

The statistical analysis was performed using SPSS version 12.0 (SPSS Inc., Chicago, IL, USA). Categorical variables were presented as means ( \pm standard deviations) and medians (interquartile ranges) with comparisons made using the chi-square and the Fisher exact test where appropriate. Data were tested for normality with the Kolmogorov-Smirnov test using the Student t-test for normally distributed data and the Kruskal-Wallis and the MannWhitney U-test for nonparametric data. A P-value of $<0.05$ was considered significant.

\section{RESULTS}

There were 168 patients presenting with $\mathrm{DE}$ plus rectal involvement who underwent elective surgery including 88 segmental rectal resections (52.3\%), 57 rectal shavings (33.9\%), and 23 discoid excisions (13.7\%). Demographic and surgical data are shown in Table 1. A PI was performed in 19 of these elective cases (11.3\%). No protective loop colostomies were used in this group. There were 4 patients $(2.3 \%)$ undergoing a Hartmann procedure and 3 patients $(1.7 \%)$ requiring a SI for postoperative complications. Additional stoma data is shown in Table 2.

The mean LOHS with uncomplicated surgery was 7.3 days (range, 3-22 days). Table 3 shows the list of postoperative complications in the cohort. There were 17 Clavien-Dindo grade III/IV complications following elective surgery (12 bowel-related and 5 urological). Of the bowel complications, there were 5 anastomotic leaks (3.0\%) and 6 rectovaginal fistulas (3.6\%). The LOHS after the diagnosis of an anastomotic leak was 25.5 days (range, 14-40 days) whereas LOHS after a rectovaginal fistula diagnosis was 15.7 days (range, 6-35 days). All of the grade III/IV bowel complications occurred after segmental rectal resections and all of the anastomotic leaks required emergency surgery.

Of the anastomotic leaks, 1 case (already with a PI) had diffuse fecal peritonitis and had the colorectal anastomosis excised and converted to an end colostomy with closure of the rectal stump. Of the 4 patients without a PI, 2 had their anastomoses dismantled with construction of a terminal colostomy. In both cases, there was a large anastomotic disruption with extensive perianastomotic soft-tissue necrosis. The other 2 patients underwent laparoscopic peritoneal lavage, drain placement, and construction of a SI.

In those patients presenting with a rectovaginal fistula, all had undergone a rectal segmental resection with colporrhaphy. In 1 case, the vagina was repaired after a hysterectomy; but in the re- 
Table 1. Demographic and surgical characteristics of the 168 patients

\begin{tabular}{|c|c|}
\hline Variable & Data \\
\hline No. of patients & 168 \\
\hline Age (yr) & $36.6 \pm 5$ \\
\hline Body mass index $\left(\mathrm{kg} / \mathrm{m}^{2}\right)$ & $21.4 \pm 9$ \\
\hline Previous surgery for endometriosis & $114(67.9)$ \\
\hline Laparoscopy & $164(97.6)$ \\
\hline Conversion & $4(2.4)$ \\
\hline \multicolumn{2}{|l|}{ Rectal resection } \\
\hline Segmental resection & $88(52.3)$ \\
\hline Discoid excision & $23(13.7)$ \\
\hline Shaving & $57(33.9)$ \\
\hline \multicolumn{2}{|l|}{ Nodule size (mm) } \\
\hline Segmental resection & $32.7 \pm 11.2$ \\
\hline Discoid excision & $23.4 \pm 10.5$ \\
\hline Shaving & $18.8 \pm 6.0$ \\
\hline \multicolumn{2}{|l|}{ Additional surgical procedure } \\
\hline Hysterectomy and salpingectomy & $77(45.8)$ \\
\hline Unilateral adnexectomy & $58(34.5)$ \\
\hline Bilateral adnexectomy & $15(8.9)$ \\
\hline Unilateral parametrial excision & $20(11.9)$ \\
\hline Bilateral parametrial excision & $14(8.3)$ \\
\hline Excision of ovarian endometrioma & $73(43.5)$ \\
\hline Unilateral ureteric reimplants & $17(10.1)$ \\
\hline Partial cystectomy & $10(5.9)$ \\
\hline Appendicectomy & $10(5.9)$ \\
\hline lleal resection & $2(1.2)$ \\
\hline
\end{tabular}

Values are presented as number only, mean \pm standard deviation, or number $(\%)$.

mainder, the vaginal repair followed a colpotomy and partial excision of an endometrioma. There were 4 patients with a rectovaginal fistula who already had a PI in place; 3 of whom were successfully managed conservatively with a combination of intravenous antibiotics and parenteral nutrition. The remaining case underwent a laparoscopic peritoneal lavage with a redo colorectal anastomosis. The 2 other patients with a rectovaginal fistula (but without an initial PI) underwent salvage surgery; 1 having construction of a SI and the other a terminal colostomy.

No association between having a PI with not developing an anastomotic leak or a rectovaginal fistula could be found $(\mathrm{P}=0.24)$.

Urological complications were diagnosed an average of 16.5 days after initial surgery (range, 15-24 days). These complications included 2 ureteric fistulas (1.2\%) and 1 ureteric stenosis (0.6\%) with all 3 complications occurring after segmental rectal resections. In each case, the injury was in the distal ureter with both ureteric fistulas associated with a difficult ureterolysis secondary
Table 2. Surgical technique associated to stoma creation

\begin{tabular}{lclcc}
\hline Surgical technique & $\mathrm{PI}$ & $\mathrm{SI}$ & Colostomy & Stoma \\
\hline Shaving & $1(3.8)$ & $0(0)$ & $0(0)$ & $1(3.8)$ \\
Discoid excision & $0(0)$ & $0(0)$ & $0(0)$ & $0(0)$ \\
Segmental resection & $18(69.2)$ & $3(11.5)$ & $4(2.4)$ & $25(96.2)$ \\
Total stomas & $19(11.3)$ & $3(1.8)$ & $4(2.4)$ & $26(15.5)$ \\
\hline
\end{tabular}

Values are presented as number (\%).

$\mathrm{PI}$, protective loop ileostomy; SI, secondary loop ileostomy.

Table 3. Postoperative complications (Clavien-Dindo classification)

\begin{tabular}{|c|c|c|}
\hline Grade & Type of complication & Management \\
\hline I & Postoperative ileus, 1 (0.6) & Nil per mouth, $1(0.6)$ \\
\hline \multirow[t]{3}{*}{$\|$} & Anemia, 2 (1.2) & Blood transfusion, 2 (1.2) \\
\hline & Urinary tract infection, $1(0.6)$ & Antibiotics, 1 (0.6) \\
\hline & Pelvic hematoma, 1 (0.6) & Antibiotics, $1(0.6)$ \\
\hline \multirow[t]{6}{*}{ III, IV } & Bowel-related & \\
\hline & $\begin{array}{l}\text { Colorectal anastomotic leak, } \\
5 \text { (3); rectovaginal fistula, } 6 \text { (3.6) }\end{array}$ & $\begin{array}{l}\text { Surgery, } 8 \text { (4.8); conservative } \\
\text { treatment, } 3(1.8)\end{array}$ \\
\hline & Rectal bleeding, $1(0.6)$ & Endoscopy, 1 (0.6) \\
\hline & Urological & \\
\hline & $\begin{array}{l}\text { Ureteric fistula, } 2 \text { (1.2); } \\
\quad \text { ureteric stenosis, } 1 \text { (0.6) }\end{array}$ & $\begin{array}{l}\text { Surgery, } 1 \text { (0.6); stent, } 1 \text { (0.6); } \\
\text { nephrostomy, } 1(0.6)\end{array}$ \\
\hline & Hemoperitoneum, 2 (1.2) & Surgical drainage, 2 (1.2) \\
\hline
\end{tabular}

Values are presented as number (\%).

to infiltrative endometriosis. One of these fistulas was successfully managed with a temporary stent but the other patient required a neoureterocystostomy. The patient with the ureteric stenosis had previously undergone a partial cystectomy with a neoureterocystostomy. This case was managed with a stent and a nephrostomy both of which were able to be removed after 6 months with complete resolution of the stenosis. Of the Clavien-Dindo grade III/IV complications, there was 1 other patient who presented 24 hours after a discoid excision with rectal bleeding and who was successfully managed endoscopically. There were 2 additional cases after segmental resection who presented with hemoperitoneum that were successfully managed in both cases by conservative means.

\section{Ostomy construction and closure}

There were 26 ostomies (15.5\%) in total constructed in 25 patients including 19 PIs, 3 SIs, and 4 end colostomies. There were no emergency room visits or hospital readmissions for any ostomy-related complications. Prior to stoma closure, anastomosis integrity was assessed using a barium enema in 13 (50.0\%), endoscopy in 10 (38.5\%), and a CT scan as the principal determinant in 1 case (3.8\%). In 1 patient (3.8\%), severe anastomotic stenosis was detected and 2 endoscopic dilatations were required before successful ileostomy closure. 
All 22 temporary ileostomies (19 PIs and 3 SIs) were reversed. Where recovery from the initial DE surgery was uncomplicated, the median time to PI closure was 5.8 months (range, 0.4-16.7 months) as compared with 9.2 months (range, 4.7-18.4 months) in those patients where postoperative complications were recorded $(\mathrm{P}=0.019)$. The mean operative time for ileostomy closure was 62 minutes (range, 45-90 minutes), and the mean LOHS after ileostomy closure was 8.3 days (range, 6-17 days). No difference was noted in the mean LOHS after stoma closure between patients with or without an initial postoperative complication $(8.8 \pm 2.59$ days vs. $8.06 \pm 2.62$ days, respectively; $\mathrm{P}=0.350$ ). Three of the 4 patients with an end colostomy were successfully reversed with the final case who developed a rectovaginal fistula following a Hartmann procedure left with a permanent colostomy. Therefore, there was a $15 \%$ chance of needing a stoma for $\mathrm{DE}$ with rectal involvement with a $4 \%$ risk once a stoma was created that it would be permanent and a $0.6 \%$ risk overall of a permanent stoma.

\section{DISCUSSION}

This article details the outcome of 168 cases of DE involving the rectum presented over a decade to a single center and managed by a multidisciplinary team. The bowel procedures performed included rectal shaves, disc excisions, and segmental resections with 296 ancillary operations, the vast majority of which were successfully conducted laparoscopically. PIs were used in $11.3 \%$ of cases but with 26 ostomies (15.5\%) used in management of the total patient group. There were 17 Clavien-Dindo grade III/IV complications in the cohort with 5 anastomotic leaks (3\%), 6 rectovaginal fistulas (3.6\%), and 3 ureteric fistulas/stenoses (1.8\%). All patients with a bowel complication were diverted with 1 patient in the entire cohort ( $4 \%$ of all stomas constructed) left with a permanent stoma. The median time to stoma closure was greater in those patients with postoperative complications when compared to those whose postoperative course was uncomplicated.

Our retrospective analysis shows the safety of selective surgical therapy in DE with rectal involvement and these results are in keeping with other reports $[3,4,11]$. The surgical decisions amount to a choice of a radical procedure eliminating all implants combined with segmental bowel resection or for more conservative surgery consisting of disc excisions and shaves. Such decisionmaking is largely dependent upon the size and extent of endometriotic deposits with the aim to improve the quality of life of the patient, to delay recurrence, and where possible to optimize fertility. The aim of our study was to assess the value of protective ileostomies and stoma use overall along with the stoma-related outcome in this unselected group of patients.

In general, patients who undergo elective surgery for endometriosis with rectal involvement have a relatively low risk for anastomotic leakage when compared with older patients who have bowel resections for other reasons. Cancer patients undergoing a rectal resection and where the decision is made for a PI are not strictly comparable since they typically can have significantly associated comorbidities and may have undergone neoadjuvant therapy. Each of these factors is major contributor to an increased risk for an anastomotic leak and for the choice of a concomitant PI, particularly where a low or ultra-low rectal resection has been performed. A PI is more commonly recommended, however, for patients undergoing multivisceral resections, representing a point of similarity between some cancer and DE patients [16]. The decision for diversion is always a balance between the likely benefit conferred by a stoma and the impact on the patient's quality of life and physical and mental well-being that is imposed by construction of an ostomy and also by its future closure [17].

Our rate of utilization of a PI for elective DE is in keeping with other studies where it has ranged between 3.9\% and 30\% overall [18]. The findings from our study serve to assist in counseling patients providing them with the knowledge that although secondary diversion may well be needed if a significant postoperative complication occurs, that there is also a high likelihood that a SI or end colostomy will ultimately be reversed. Such information allays some of the treatment fears and creates a platform of patient education concerning temporary stoma use which has the potential to positively impact LOHS and reduce the likelihood for hospital readmission [19]. This sort of information is also of particular value when stoma closure may be delayed as occurred in some of our cases with a major postoperative complication. Although cancer patients with longer-term stomas are not exactly comparable it is evident that a significant delay in closure of a temporary ostomy appears to have a relatively little negative impact on global quality of life parameters [20].

Recently, Vigueras Smith et al. [21] conducted a literature review that included retrospective studies and systematic analyses specifically addressing the issue of anastomotic leakage following DE surgery. These authors provided general recommendations for temporary bowel diversion in those deemed at higher risk of anastomotic leakage. This study took into account the distance of the anastomosis from the anal verge, the size of any endometriotic nodule that was excised, and those cases where a vaginal resection was concomitantly performed. The decision for a PI in our unit is principally based upon the level of the anastomosis, the presence of potentially correctable preoperative risk factors (such as anemia, malnutrition, smoking, alcohol consumption, steroids, and a high body mass index), and the need for more complex surgery (hysterectomy, colpotomy, and/or neoureterocystostomy). It is appreciated that strict comparisons between our results and others are difficult as patients are not matched for disease extent and severity. However, similar to our results, Abo et al. [18] reported more Clavien-Dindo grade III/IV complications following segmental resection than with alternate procedures. It presently remains unclear whether shaving can be utilized more often in extensive disease in order to avoid these severe postoperative complications.

We noted a $3.6 \%$ incidence of rectovaginal fistula in our pa- 
tients. This rate is similar to that reported by Abo et al. [18] where there were 14 of 364 of the cases (3.8\%) and also by Kondo et al. [22] where there were only 8 of 568 of the cases (1.4\%). Results are difficult to compare as differences in the ages of patients, the size of endometriotic nodules, the use of a disc excision for vaginal infiltration, and the number of prior surgeries for endometriosis will all affect the risk for a rectovaginal fistula. Beyond the cystoscopic instillation of ICG to demarcate the ureters [10] lately we have been using intravenous ICG in the predictive assessment of anastomotic viability [23] with the potential that this approach could influence the decision for a PI in selected cases. Intravenous ICG also has a demonstrated capacity to delineate pelvic endometriotic lesions [24] where Raimondo et al. [25] have recently shown that some of the larger deposits of endometriosis have a hypovascular fluorescence pattern that is distinguishable from normal tissue.

It is accepted that a PI will not prevent an anastomotic leak, but rather will diminish the consequences of leakage [26]. According to literature, we could not find an association between having a PI and not developing an anastomotic leak o or a rectovaginal fistula $(\mathrm{P}=0.240)$. This result has to be carefully analyzed, as our PI cohort $(n=19)$ is too small to draw strong conclusions. Early diagnosis of postoperative anastomotic leak is essential in order to promptly establish the most appropriate treatment. In most cases, a successful outcome can be achieved with laparoscopic intervention although this depends upon the type and extent of peritonitis [27]. When an anastomotic leak occurred without fecal peritonitis, it was sufficient to perform a laparoscopic lavage with proximal diversion using an SI. In this respect, the SI is favored over a protective colostomy because of ease of construction and closure and because of a lower rate of stoma prolapse and parastomal hernia [28]. The use of an SI permits a simple stomal closure later on and it is more readily constructed laparoscopically than a loop colostomy [29]. A Hartmann procedure with its attendant morbidity of later reversal was reserved for anastomotic leaks where there was diffuse fecal peritonitis.

Regarding urological complications, we found a rate of $1.2 \%$ ureteral fistula and $0.6 \%$ ureteral stenosis for the 168 patients, which is in accordance with the results for endometriosis surgery published by other groups [30]. The urological complication rate in patients with $\mathrm{DE}$ may be greater than in patients operated on for rectal cancer, since DE may in many cases require ureterolysis due to infiltration of the ureters. For this reason, in our unit, ureters are cannulated with indocyanine green to try to prevent these complications.

There are several limitations to our study. In particular, its retrospective nature has the potential for bias in the assessment of different procedures. In general, there were more complications of greater severity following resectional operations but these were associated with more extensive disease. Although our findings are in keeping with Abo et al. [18], we cannot from these data advocate a strategy that would prioritize rectal shaving over segmental resection for more extensive disease. The preliminary data reported from the ENDORE trial (Functional Outcomes of Surgical Management of Deep Endometriosis Infiltrating the Rectum, NCT 01291576) which randomized patients presenting with rectal endometriosis to conservative surgery or segmental resection, failed to show advantage for functional digestive or urinary outcomes with a more conservative approach [31]. This study, however, lacked sufficient statistical power to determine differences between the groups or to longitudinally assess differential rates of recurrence.

In summary, the different laparoscopic surgical options for advanced $\mathrm{DE}$ with rectal infiltration have an acceptably low morbidity. A selective approach towards a PI was reserved for low anastomoses and more extensive pelvic reconstructive surgeries. Most stomas, whether protective or when used definitively for major postoperative complications, were able to be reversed.

\section{CONFLICT OF INTEREST}

No potential conflict of interest relevant to this article was reported.

\section{ACKNOWLEDGMENTS}

We thank Andrew Zbar, M.D., Director of Complete Manuscript Services, for his critical review of the manuscript.

\section{REFERENCES}

1. Burney RO, Giudice LC. Pathogenesis and pathophysiology of endometriosis. Fertil Steril 2012;98:511-9.

2. Jenkins S, Olive DL, Haney AF. Endometriosis: pathogenetic implications of the anatomic distribution. Obstet Gynecol 1986;67: 335-8.

3. Wolthuis AM, Meuleman C, Tomassetti C, D'Hooghe T, de Buck van Overstraeten A, D'Hoore A. Bowel endometriosis: colorectal surgeon's perspective in a multidisciplinary surgical team. World J Gastroenterol 2014;20:15616-23.

4. Ruffo G, Scopelliti F, Scioscia M, Ceccaroni M, Mainardi P, Minelli L. Laparoscopic colorectal resection for deep infiltrating endometriosis: analysis of 436 cases. Surg Endosc 2010;24:63-7.

5. Donnez O, Roman $\mathrm{H}$. Choosing the right surgical technique for deep endometriosis: shaving, disc excision, or bowel resection? Fertil Steril 2017;108:931-42.

6. Roman H; FRIENDS group (French coloRectal Infiltrating ENDometriosis Study group). A national snapshot of the surgical management of deep infiltrating endometriosis of the rectum and colon in France in 2015: a multicenter series of 1135 cases. J Gynecol Obstet Hum Reprod 2017;46:159-65.

7. Akladios C, Messori P, Faller E, Puga M, Afors K, Leroy J, et al. Is ileostomy always necessary following rectal resection for deep infiltrating endometriosis? J Minim Invasive Gynecol 2015;22:103- 
9.

8. Mabrouk M, Clarizia R, Grassi T, Ruffo G, Roviglione G, Bruni F, et al. Protective ileostomy in colorectal resection for endometriosis: is it truly protective? J Minim Invasive Gynecol 2015;22(6S): S177.

9. Dindo D, Demartines N, Clavien PA. Classification of surgical complications: a new proposal with evaluation in a cohort of 6336 patients and results of a survey. Ann Surg 2004;240:205-13.

10. Mandovra P, Kalikar V, Patankar RV. Real-time visualization of ureters using indocyanine green during laparoscopic surgeries: can we make surgery safer? Surg Innov 2019;26:464-8.

11. Hernández Gutiérrez A, Spagnolo E, Zapardiel I, Garcia-Abadillo Seivane R, López Carrasco A, Salas Bolívar P, et al. Post-operative complications and recurrence rate after treatment of bowel endometriosis: comparison of three techniques. Eur J Obstet Gynecol Reprod Biol X 2019;4:100083.

12. van Rooijen SJ, Jongen AC, Wu ZQ, Ji JF, Slooter GD, Roumen $\mathrm{RM}$, et al. Definition of colorectal anastomotic leakage: a consensus survey among Dutch and Chinese colorectal surgeons. World J Gastroenterol 2017;23:6172-80.

13. Smith SR, Pockney P, Holmes R, Doig F, Attia J, Holliday E, et al. Biomarkers and anastomotic leakage in colorectal surgery: C-reactive protein trajectory is the gold standard. ANZ J Surg 2018;88: 440-4.

14. Siewert B, Tye G, Kruskal J, Sosna J, Opelka F, Raptopoulos V, et al. Impact of CT-guided drainage in the treatment of diverticular abscesses: size matters. AJR Am J Roentgenol 2006;186:680-6.

15. Bonin E, Bridoux V, Chati R, Kermiche S, Coget J, Tuech JJ, et al. Diverting stoma-related complications following colorectal endometriosis surgery: a 163-patient cohort. Eur J Obstet Gynecol Reprod Biol 2019;232:46-53.

16. Shiomi A, Ito M, Saito N, Hirai T, Ohue M, Kubo Y, et al. The indications for a diverting stoma in low anterior resection for rectal cancer: a prospective multicentre study of 222 patients from Japanese cancer centers. Colorectal Dis 2011;13:1384-9.

17. Siassi M, Hohenberger W, Lösel F, Weiss M. Quality of life and patient's expectations after closure of a temporary stoma. Int J Colorectal Dis 2008;23:1207-12.

18. Abo C, Moatassim S, Marty N, Saint Ghislain M, Huet E, Bridoux $\mathrm{V}$, et al. Postoperative complications after bowel endometriosis surgery by shaving, disc excision, or segmental resection: a threearm comparative analysis of 364 consecutive cases. Fertil Steril 2018;109:172-8

19. Danielsen AK, Burcharth J, Rosenberg J. Patient education has a positive effect in patients with a stoma: a systematic review. Colorectal Dis 2013;15:e276-83.

20. Herrle F, Sandra-Petrescu F, Weiss C, Post S, Runkel N, Kienle P.
Quality of life and timing of stoma closure in patients with rectal cancer undergoing low anterior resection with diverting stoma: a multicenter longitudinal observational study. Dis Colon Rectum 2016;59:281-90.

21. Vigueras Smith A, Sumak R, Cabrera R, Kondo W, Ferreira H. Bowel anastomosis leakage following endometriosis surgery: an evidence based analysis of risk factors and prevention techniques. Facts Views Vis Obgyn 2020;12:207-25.

22. Kondo W, Bourdel N, Tamburro S, Cavoli D, Jardon K, Rabischong $\mathrm{B}$, et al. Complications after surgery for deeply infiltrating pelvic endometriosis. BJOG 2011;118:292-8.

23. De Nardi P, Elmore U, Maggi G, Maggiore R, Boni L, Cassinotti E, et al. Intraoperative angiography with indocyanine green to assess anastomosis perfusion in patients undergoing laparoscopic colorectal resection: results of a multicenter randomized controlled trial. Surg Endosc 2020;34:53-60.

24. Ianieri MM, Della Corte L, Campolo F, Cosentino F, Catena U, Bifulco G, et al. Indocyanine green in the surgical management of endometriosis: a systematic review. Acta Obstet Gynecol Scand 2021;100:189-99.

25. Raimondo D, Mastronardi M, Mabrouk M, Cafagna G, Salucci P, Arena A, et al. Rectosigmoid endometriosis vascular patterns at intraoperative indocyanine green angiography and their correlation with clinicopathological data. Surg Innov 2020;27:474-80.

26. Wong NY, Eu KW. A defunctioning ileostomy does not prevent clinical anastomotic leak after a low anterior resection: a prospective, comparative study. Dis Colon Rectum 2005;48:2076-9.

27. Wind J, Koopman AG, van Berge Henegouwen MI, Slors JF, Gouma DJ, Bemelman WA. Laparoscopic reintervention for anastomotic leakage after primary laparoscopic colorectal surgery. Br J Surg 2007;94:1562-6.

28. Rondelli F, Reboldi P, Rulli A, Barberini F, Guerrisi A, Izzo L, et al. Loop ileostomy versus loop colostomy for fecal diversion after colorectal or coloanal anastomosis: a meta-analysis. Int J Colorectal Dis 2009;24:479-88.

29. Gavriilidis P, Azoulay D, Taflampas P. Loop transverse colostomy versus loop ileostomy for defunctioning of colorectal anastomosis: a systematic review, updated conventional meta-analysis, and cumulative meta-analysis. Surg Today 2019;49:108-17.

30. Cunha FL, Arcoverde FV, Andres MP, Gomes DC, Bautzer CR, Abrao MS, et al. Laparoscopic treatment of ureteral endometriosis: a systematic review. J Minim Invasive Gynecol 2021;28:77987.

31. Roman H, Bubenheim M, Huet E, Bridoux V, Zacharopoulou C, Daraï $\mathrm{E}$, et al. Conservative surgery versus colorectal resection in deep endometriosis infiltrating the rectum: a randomized trial. Hum Reprod 2018;33:47-57. 